\title{
The quasi-nonassociative exceptional $F(4)$ deformed quantum oscillator
}

\author{
N. Aizawa, Z. Kuznetsova ${ }^{\dagger}$ and F. Toppan ${ }^{\ddagger}$ \\ November 22, 2017 \\ * Department of Physical Science, Graduate School of Science, \\ Osaka Prefecture University, Nakamozu Campus, Sakai, Osaka 599-8531 Japan. \\ $\dagger U F A B C, A v$. dos Estados 5001, Bangu, \\ cep 09210-580, Santo André (SP), Brazil. \\ ‡ CBPF, Rua Dr. Xavier Sigaud 150, Urca, \\ cep 22290-180, Rio de Janeiro (RJ), Brazil.
}

\begin{abstract}
We present the deformed (for the presence of Calogero potential terms) onedimensional quantum oscillator with the exceptional Lie superalgebra $F(4)$ as spectrum-generating superconformal algebra. The Hilbert space is given by a 16-ple of square-integrable functions. The energy levels are $\frac{2}{3}+n$, with $n=0,1,2, \ldots$. The ground state is 7 times degenerate. The excited states are 8 times degenerate. The $(7,8,8,8, \ldots)$ semi-infinite tower of states is recovered from the $(7 ; 8 ; 1)$ supermultiplet of the $\mathcal{N}=8$ worldline supersymmetry. The model is unique, up to similarity transformations, and admits an octonionic-covariant formulation which manifests itself as "quasi-nonassociativity". This means, in particular, that the Calogero coupling constants are expressed in terms of the octonionic structure constants.

The associated $F(4)$ superconformal quantum mechanics is also presented.
\end{abstract}

CBPF-NF-002/17

*E-mail: aizawa@p.s.osakafu-u.ac.jp

${ }^{\dagger} \mathrm{E}-\mathrm{mail}:$ zhanna.kuznetsova@ufabc.edu.br

${ }^{\ddagger}$ E-mail: toppan@cbpf.br 


\section{Introduction}

We present the unique (up to similarity transformations) one-dimensional superconformal quantum mechanical system with the $F(4)$ Lie superalgebra as dynamical symmetry; its associated (via the de Alfaro-Fubini-Furlan construction [1]) deformed (due to the presence of Calogero's [2] potential terms) quantum oscillator, is also derived. The Hilbert space of the latter model and its discrete, bounded from below, spectrum is obtained.

The division algebra of the octonions are at the core of several exceptional structures in mathematics. The 5 exceptional Lie algebras are all related to the octonions. Indeed $g_{2}$ is the Lie algebra of the $G_{2}$ group of automorphisms of the octonions, while the remaining 4 exceptional Lie algebras, $f_{4}, e_{6}, e_{7}$ and $e_{8}$, are induced by the octonions via the Freudenthal-Tits magic square construction. About simple Lie superalgebras, the exceptional Lie superalgebras $G(3)$ and $F(4)$ entering the Kac's classification [3] admit an octonionic realization [4].

We demanded octonionic covarariance as a key ingredient to derive the $F(4)$ models. This means that the differential operators of the spectrum-generating superalgebra are expressed in terms of the octonionic structure constants.

We obtained at first the most general, octonionic covariant and scale invariant, $\mathcal{N}=8$ supersymmetric quantum mechanics defined on 8 bosonic and 8 fermionic fields. We further implemented the superconformal constraint which selects a critical theory with enhanced symmetry. The existence, at the critical value, of 7 linear constraints for the $28 R$-symmetry generators, unambiguously determines $F(4)$ (whose $R$-symmetry is so( 7 ), with $21=28-7$ generators) as the dynamical symmetry superconformal algebra of the model.

The model is characterized by "quasi-nonassociativity". The Calogero's coupling constants are expressed in terms of the octonionic structure constants $C_{i j k}$ which encode the nonassociativity of the octonions.

Octonionic covariance was used in [5] to construct the most general classical $\mathcal{N}=8$ extended supersymmetric one-dimensional sigma-model in the Lagrangian framework based on the $(1 ; 8 ; 7)$ irreducible supermultiplet, see [6], of the $\mathcal{N}=8$ worldline supersymmetry. It was proved in [7] that an $F(4)$ superconformally invariant, one-dimensional sigma-model results from a suitable restriction of the parameters entering the $\mathcal{N}=8,(1 ; 8 ; 7)$ theory. In [8] and [9] the systematic construction of one-dimensional superconformal algebras in terms of worldline supermultiplets ( $D$-module representations) was obtained. We recall that one-dimensional superconformal algebras are simple Lie superalgebras satisfying a class of restrictions, resulting from their possible interpretation as dynamical symmetries of superconformal mechanics. Here it is sufficient to remind that the even sector should be decomposed into a direct $\operatorname{sum} \operatorname{sl}(2) \oplus R$, with $R$ known as the $R$-symmetry algebra, while the odd sector is decomposed into a set of $\mathcal{N}+\mathcal{N}$, dually related generators.

From [8, 9] follows, in particular, that octonionic-covariant $D$-module realizations are encountered at $\mathcal{N}=7$ for the exceptional $G(3)$ superalgebra and at $\mathcal{N}=8$ for the superalgebras $D(4,1) \approx \operatorname{osp}(8 \mid 2)$ and $F(4)$.

It was noted in [10] that "trigonometric" $D$-module representations allow the construction of worldline sigma-models which correspond to a classical (and superconformal) version of the de Alfaro-Fubini-Furlan prescription. 
The quantization of the interacting trigonometric models was first obtained in [11], by applying standard techniques to pass from the classical Lagrangian to the quantum Hamiltonian formulation. In particular deformed quantum oscillators with $D(2,1 ; \alpha)$ as spectrum-generating superalgebras were derived.

The method used in this paper allows to directly construct a quantum mechanical system, bypassing the scheme of deriving at first a classical Lagrangian model which is later quantized in the Hamiltonian framework. For this reason we did not need to introduce a classical Lagrangian trigonometric version (which has not been constructed, yet) of the classical $F(4)$ one-dimensional sigma-model introduced in [7].

We postpone to the Conclusions a further discussion about the results of our model and about some issues of nonassociativity in physics.

The scheme of the paper is as follows. In Section $\mathbf{2}$ we introduce the octonioniccovariant formulation. In Section 3 we derive the octonionic-covariant, scale invariant, $\mathcal{N}=8$ supersymmetric quantum mechanics. In Section 4 we derive the differential realization of the $F(4)$ Lie superalgebra. The deformed $F(4)$ oscillator is introduced in Section 5. In Section 6 the spectrum of the theory is obtained and the quasi-nonassociativity of the model is discussed. In the Conclusions we comment about our results and the issue of nonassociativity in physics.

\section{Octonions and the octonionic covariance}

The octonionic multiplication is encoded in the basic relations, for the seven imaginary octonions $e_{i}(i=1,2, \ldots, 7)$,

$$
e_{i} e_{j}=-\delta_{i j}+C_{i j k} e_{k}
$$

Here and in the following, unless otherwise specified, the sum over repeated indices is

understood. $C_{i j k}$ is the totally antisymmetric octonionic structure constant. Besides the rank $3 C_{i j k}$ tensor, two more totally antisymmetric constant tensors (of rank 4 and 7) are compatible with the octonionic multiplication; they are given by $C_{i j k l}$ and $\epsilon_{i j k l m n p}$. We assume the rank 3, 4, 7 totally antisymmetric tensors to be normalized according to:

$$
\begin{gathered}
C_{123}=C_{147}=C_{165}=C_{246}=C_{257}=C_{354}=C_{367}=1, \\
C_{4567}=C_{2356}=C_{2437}=C_{1357}=C_{1346}=C_{1276}=C_{1245}=1, \\
\epsilon_{1234567}=1 .
\end{gathered}
$$

Due to a relation involving the three totally antisymmetric constant tensors, only two of them are independent. The relation can be expressed as

$$
6 C_{i j k l}=\epsilon_{i j k l m n p} C_{m n p}
$$

The seven imaginary octonions can be conveniently arranged in the famous Fano's projective plane (see [12] for a review). The non-vanishing $C_{i j k}$ 's correspond to three points belonging to one of its seven lines. The non-vanishing $C_{i j k l}$ 's correspond to the four points which are complementary to each one of the seven lines. 
The octonions induce a realization of the Clifford algebra $C l(0,7)$ based on the following construction. Let $x=x_{0}+x_{j} e_{j}$ be a real octonion, parametrized by eight real numbers $x_{0}, x_{j} \in \mathbb{R}$, so that $\vec{x}=\left(x_{0}, x_{j}\right)^{T}$ is an 8-component real vector. The mapping

$$
x \mapsto e_{i} x=x_{i}^{\prime}
$$

can be expressed as a linear transformation

$$
\vec{x}_{i}^{\prime}=\gamma_{i} \vec{x} .
$$

It is easily proved that the seven $8 \times 8$ matrices $\gamma_{i}$ so induced satisfy the $C l(0,7)$ Clifford algebra fundamental relation

$$
\gamma_{i} \gamma_{j}+\gamma_{j} \gamma_{i}=-2 \delta_{i j} \mathbb{I}_{8}, \quad i, j=1,2, \ldots, 7
$$

(here and in the following $\mathbb{I}_{n}$ denotes the $n \times n$ identity matrix).

Furthermore, their entries are expressed in terms of the octonionic structure constants $C_{i j k}$ according to

$$
\left(\gamma_{i}\right)_{L M}=\left(\begin{array}{c|c}
0 & \delta_{i m} \\
\hline-\delta_{i l} & C_{i l m}
\end{array}\right)
$$

where $L, M$ take values $L=0, l$ and $M=0, m$, with $l, m=1,2, \ldots, 7$. The (77) matrices are obtained, up to an overall sign, from the (4) map.

The double role played by $C_{i j k}$ should be duly noted. Entering (1) it is responsible for the non-associativity of the octonionic multiplication. We have, e.g.:

$$
\left(e_{1} e_{2}\right) e_{4}=e_{3} e_{4}=-e_{5} \neq e_{1}\left(e_{2} e_{4}\right)=e_{1} e_{6}=e_{5} .
$$

On the other hand $C_{i j k}$ enters (6) as well, providing a matrix realization for the associative $C l(0,7)$ Clifford algebra. One can say that, in this matrix realization, $C_{i j k}$ encodes the remnant of the non-associativity of the octonions.

The $C l(0,7)$ Clifford algebra gives a basis for the 64 -dimensional vector space of $8 \times 8$ real matrices. Schematically, the elements of given $\operatorname{rank} r=0,1,2,3$ (entering $\gamma^{(r)}$ ) are

$$
\begin{aligned}
\gamma^{(0)} & \equiv \mathbb{I}_{8}, \\
\gamma^{(1)} & \equiv \gamma_{i}, \\
\gamma^{(2)} & \equiv \gamma_{i} \gamma_{j} \quad(i<j), \\
\gamma^{(3)} & \equiv \gamma_{i} \gamma_{j} \gamma_{k} \quad(i<j<k) .
\end{aligned}
$$

Due to Hodge duality, the product of $7-r$ different matrices $\gamma_{i}$ is equivalent to the product of matrices of rank $r$. There is a total number of $\left(\begin{array}{l}7 \\ r\end{array}\right)$ matrices of rank $r$, so that

$$
\left(\begin{array}{l}
7 \\
0
\end{array}\right)+\left(\begin{array}{l}
7 \\
1
\end{array}\right)+\left(\begin{array}{l}
7 \\
2
\end{array}\right)+\left(\begin{array}{l}
7 \\
3
\end{array}\right)=1+7+21+35=64
$$

$\gamma^{(0)}$ and $\gamma^{(3)}$ provide the basis for the 36 symmetric $8 \times 8$ matrices, while $\gamma^{(1)}$ and $\gamma^{(2)}$ provide the basis for the 28 antisymmetric $8 \times 8$ matrices. 
The introduction of supersymmetry requires acting on a $\mathbb{Z}_{2}$-graded vector space of even and odd elements (identified with bosons and fermions), with block-antidiagonal supersymmetry operators. In the present case this can be obtained by doubling the size of the vector space by introducing the nine $16 \times 16$ matrix generators of the $C l(9,0)$ Clifford algebra $\Gamma_{A}(A=1,2, \ldots, 8,9)$ through the positions

$$
\Gamma_{i}=\left(\begin{array}{cc}
0 & \gamma_{i} \\
-\gamma_{i} & 0
\end{array}\right), \quad \Gamma_{8}=\left(\begin{array}{cc}
0 & \mathbb{I}_{8} \\
\mathbb{I}_{8} & 0
\end{array}\right), \quad \Gamma_{9}=\left(\begin{array}{cc}
\mathbb{I}_{8} & 0 \\
0 & -\mathbb{I}_{8}
\end{array}\right) .
$$

In the following different symbols are employed for different ranges of values: $i, j=$ $1,2, \ldots, 7$, while $I, J=1,2, \ldots, 8$ and $A, B=1,2, \ldots 9$.

We have, by construction,

$$
\Gamma_{A} \Gamma_{B}+\Gamma_{B} \Gamma_{A}=2 \delta_{A B} \mathbb{I}_{16}
$$

The block-diagonal matrix $\Gamma_{9}$ can now be identified with the Fermion Parity Operator of the Supersymmetric Quantum Mechanics. Its \pm 1 eigenvalues determine the 8-dimensional bosonic $(+1)$ and fermionic $(-1)$ vector spaces.

The 256-dimensional vector space of $16 \times 16$ real matrices can be expressed in terms of the $C l(9,0)$ Clifford algebra matrix generators $\Gamma_{A}$ 's. The different $\left(\begin{array}{l}9 \\ \mathbf{r}\end{array}\right)$ rank $\mathbf{r}$ tensors are compactly written as $\Gamma^{(\mathbf{r})} \equiv \Gamma_{A_{1}} \ldots \Gamma_{A_{\mathbf{r}}}$ for $A_{1}<A_{2}<\ldots<A_{\mathbf{r}}(\mathbf{r}=0,1,2,3,4)$. The analogue of formula (9) now reads

$$
\left(\begin{array}{l}
9 \\
0
\end{array}\right)+\left(\begin{array}{l}
9 \\
1
\end{array}\right)+\left(\begin{array}{l}
9 \\
2
\end{array}\right)+\left(\begin{array}{l}
9 \\
3
\end{array}\right)+\left(\begin{array}{l}
9 \\
4
\end{array}\right)=1+9+36+84+126=256 .
$$

Due to the different status of $\Gamma_{9}$ (which is block-diagonal) with respect to the 8 remaining $\Gamma_{I}$ 's (which are block-antidiagonal), a more refined decomposition singles out $\Gamma_{9}$. A further refinement singles out $\Gamma_{8}$ (which is scalar with respect to the octonionic imaginary index) from the seven $\Gamma_{i}$ 's which carry the vectorial index $i$ associated to the imaginary octonions. Taking into account this $7+1+1$ decomposition, we arrive at the following table. The second column denotes the block diagonal $(d g)$ versus the block antidiagonal $(a d)$ character of the matrices; the third column denotes their symmetry $(S Y M)$ versus antisymmetry $(A S)$ property; the last column indicates their total number $N_{b}$. The symbol $\Gamma^{(r)}$ (for $r$ not in boldface font) denotes $\Gamma^{(r)} \equiv \Gamma_{i_{1}} \ldots \Gamma_{i_{r}}$ for $i_{1}<i_{2}<\ldots<i_{r}$, 
with $i_{r}$ ranging from 1 to 7 . We have

\begin{tabular}{|c|c|c|c|}
\hline & $d g / a d$ & $S Y M / A S$ & $N_{b}$ \\
\hline $\mathbb{I}$ & $d g$ & $S Y M$ & 1 \\
\hline$\Gamma^{(1)}$ & $a d$ & $S Y M$ & 7 \\
$\Gamma_{8}$ & $a d$ & $S Y M$ & 1 \\
$\Gamma_{9}$ & $d g$ & $S Y M$ & 1 \\
\hline$\Gamma^{(2)}$ & $d g$ & $A S$ & 21 \\
$\Gamma^{(1)} \Gamma_{8}$ & $d g$ & $A S$ & 7 \\
$\Gamma^{(1)} \Gamma_{9}$ & $a d$ & $A S$ & 7 \\
$\Gamma_{8} \Gamma_{9}$ & $a d$ & $A S$ & 1 \\
\hline$\Gamma^{(3)}$ & $a d$ & $A S$ & 35 \\
$\Gamma^{(2)} \Gamma_{8}$ & $a d$ & $A S$ & 21 \\
$\Gamma^{(2)} \Gamma_{9}$ & $d g$ & $A S$ & 21 \\
$\Gamma^{(1)} \Gamma_{8} \Gamma_{9}$ & $d g$ & $A S$ & 7 \\
\hline$\Gamma^{(4)}$ & $d g$ & $S Y M$ & 35 \\
$\Gamma^{(3)} \Gamma_{8}$ & $d g$ & $S Y M$ & 35 \\
$\Gamma^{(3)} \Gamma_{9}$ & $a d$ & $S Y M$ & 35 \\
$\Gamma^{(2)} \Gamma_{8} \Gamma_{9}$ & $a d$ & $S Y M$ & 21 \\
\hline
\end{tabular}

\section{Scale-invariant $\mathcal{N}=8$ Supersymmetric Quantum Mechanics}

We are now in the position to introduce the $\mathcal{N}=8$ Supersymmetric Quantum Mechanics, defined by the (anti)commutators

$$
\begin{aligned}
\left\{Q_{I}, Q_{J}\right\} & =2 \delta_{I J} H \\
{\left[H, Q_{I}\right] } & =0 .
\end{aligned}
$$

The eight supersymmetry operators $Q_{I}$ are Hermitian and block-antidiagonal. $H$ is a Hamiltonian which can be expressed as

$$
H=-\frac{1}{2} \partial_{x}^{2} \mathbb{I}+V(x),
$$

where $V(x)$ is a block-diagonal, real, symmetric matrix potential.

Since we are interested in Superconformal Quantum Mechanics (and the oscillator models possessing the associated superalgebra as dynamical symmetry), we investigate at first the condition to obtain scale-invariant Supersymmetric Quantum Mechanics. It follows, in particular, that the potential $V(x)$ should be expressed as

$$
V(x)=\frac{1}{x^{2}} V,
$$

where $V$ is a block-diagonal, constant, symmetric $\left(V^{T}=V\right)$ matrix.

For the moment we are working with $16 \times 16$ real matrices. It makes sense to express the differential part entering $Q_{I}$ 's to be proportional to $\Gamma_{I} \Gamma_{9} \partial_{x}$. This set of operators 
indeed satisfies the requirements of being Hermitian and block-antidiagonal. Concerning the potential terms, proportional to $\frac{1}{x}$, we assume the most general ones satisfying the criteria of being block-antidiagonal, symmetric and octonionic covariant. Under these assumptions we propose the following Ansatz for $Q_{8}$ and $Q_{i}$ 's:

$$
\begin{array}{rlrl}
Q_{8} & =\frac{1}{\sqrt{2}}\left(\Gamma_{8} \Gamma_{9} \partial_{x}+\frac{1}{x} E_{8}\right), & E_{8} & =a C_{i j k} \Gamma_{i} \Gamma_{j} \Gamma_{k} \Gamma_{9}+b \Gamma_{8}, \\
Q_{i} & =\frac{1}{\sqrt{2}}\left(\Gamma_{i} \Gamma_{9} \partial_{x}+\frac{1}{x} E_{i}\right), & E_{i}=c C_{i j k} \Gamma_{j} \Gamma_{k} \Gamma_{8} \Gamma_{9}+d C_{i j k l} \Gamma_{j} \Gamma_{k} \Gamma_{l} \Gamma_{9}+e \Gamma_{i},
\end{array}
$$

where the constants $a, b, c, d, e$ are real coefficients to be determined by the closure of (15)).

The requirement $\left\{Q_{i}, Q_{j}\right\}=0$ for $i \neq j$ is solved for either

$$
d=\frac{1}{3} c, \quad e=-\frac{1}{2}+6 c,
$$

or

$$
d=-\frac{1}{3} c, \quad e=\frac{1}{2}+6 c .
$$

Both restrictions produce a Hamiltonian $H$, invariant under $\mathcal{N}=7$ supersymmetries, possessing a diagonal potential. The constant matrix $V=\operatorname{diag}\left(v_{1}, v_{2}, v_{3}, \ldots, v_{16}\right)$ is given, in case (19) by

$$
v_{1}=\ldots=v_{8}=-\frac{1}{8}+32 c^{2}, \quad v_{9}=\frac{3}{8}+8 c+32 c^{2}, \quad v_{10}=\ldots=v_{16}=\frac{3}{8}-8 c+32 c^{2} .
$$

In case (20) $V$ is given by

$$
v_{1}=\frac{3}{8}-8 c+32 c^{2}, \quad v_{2}=\ldots=v_{8}=\frac{3}{8}+8 c+32 c^{2}, \quad v_{9}=\ldots=v_{16}=-\frac{1}{8}+32 c^{2} .
$$

A scale-invariant $\mathcal{N}=8$ Supersymmetric Quantum Mechanics is recovered by further setting, in both cases,

$$
a=-\frac{1}{3} c, \quad b=e .
$$

The scale-invariant Hamiltonians $H$, with matrix potentials determined by either (21) or (22), are $\mathcal{N}=8$ supersymmetric. They depend on an arbitrary real coupling constant $c$.

\section{The superconformal algebra realization}

The introduction of a superconformal algebra requires the presence of eight operators $\widetilde{Q}_{I}$, the superconformal partners of the supercharges $Q_{I}$. They have to be block-antidiagonal and, for dimensional reason, proportional to $x$. The anticommutators $\left\{Q_{I}, \widetilde{Q}_{J}\right\}$ should produce the dilatation operator $D$ (from $I=J$ ) and the $R$-symmetry generators (from $I \neq J)$. The dilatation operator $D$ should contain a term proportional to $x \partial_{x} \mathbb{I}$. This requirement rules out the most natural choice for $\widetilde{Q}_{I}$, consisting in setting $\widetilde{Q}_{I} \propto x \Gamma_{I}$. The anti-Hermitian choice $\widetilde{Q}_{I} \propto x \Gamma_{I} \Gamma_{9}$, on the other hands, nicely works. In order to have 
Hermitian operators $\widetilde{Q}_{I}$, we therefore need to introduce the imaginary unit $i$. It follows that, while scale-invariant $\mathcal{N}=8$ Supersymmetric Quantum Mechanics can be realized on 8 real bosonic and 8 real fermionic fields, in order to have $\mathcal{N}=8$ Superconformal Quantum Mechanics, we need to double (counting in real components) the number of fields, acting on 8 complex bosonic and 8 complex fermionic fields.

Conveniently normalized, the operators $\widetilde{Q}_{I}$ 's are chosen to be

$$
\widetilde{Q}_{I}=\frac{i}{\sqrt{2}} x \Gamma_{I} \Gamma_{9}
$$

They satisfy the anticommutators

$$
\left\{\widetilde{Q}_{I}, \widetilde{Q}_{J}\right\}=2 \delta_{I J} K
$$

where

$$
K=\frac{1}{2} x^{2} \mathbb{I}_{16}
$$

The next topic consists in investigating the anticommutators $\left\{Q_{I}, \widetilde{Q}_{J}\right\}$ and determinining under which conditions (if any) an $\mathcal{N}=8$ superconformal algebra is recovered.

At any given $I$ we have

$$
\left\{Q_{I}, \widetilde{Q}_{I}\right\}=-i\left(x \partial_{x}+\frac{1}{2}\right) \mathbb{I}_{16}:=D
$$

Therefore, without loss of generality, we can set

$$
\left\{Q_{I}, \widetilde{Q}_{J}\right\}=\delta_{I J} D+R_{I J},
$$

where, for $I \neq J$,

$$
R_{I J}=\frac{i}{2}\left(-\Gamma_{I} \Gamma_{J}+\left\{E_{I}, \Gamma_{J} \Gamma_{9}\right\}\right)
$$

We recall that the operators $E_{I}$ have been introduced in (18). Either the choice (19)23) or the choice (20123) of the real parameters have been assumed. For both choices, the $R_{I J}$ 's turn out to be antisymmetric with respect to the $I \leftrightarrow J$ exchange:

$$
R_{I J}=-R_{J I}
$$

In order to guarantee the closure, as Lie superalgebra, of the set of generators $H, D, K, Q_{I}, \widetilde{Q}_{I}, R_{I J}$, we need to check under which condition the $R_{I J}$ 's operators form a closed ( $R$-symmetry) Lie algebra and the fermionic operators $Q_{I}$ 's $\left(\widetilde{Q}_{I}\right.$ 's $)$ belong to an $R$-symmetry representation.

These two requirements select specific values for $c$ entering (18). With the choice (19) for $d, e$, the value of $c$ is fixed to be $c=\frac{1}{12}$. Taking into account (23), the parameters $a, b, c, d, e$ entering (18) are thus determined to be

$$
a=-d=-\frac{1}{36}, \quad c=\frac{1}{12}, \quad b=e=0 .
$$


With the choice (20) for $d, e$, the selected value of $c$ is $c=-\frac{1}{12}$. The parameters $a, b, c, d, e$ entering (18) are given in this case by

$$
a=d=\frac{1}{36}, \quad c=-\frac{1}{12}, \quad b=e=0 .
$$

The two choices turn out to be equivalent. In the first case the potential is expressed, see (21), by

$$
v_{1}=\ldots=v_{8}=\frac{7}{72}, \quad v_{9}=\frac{91}{72}, \quad v_{10}=\ldots=v_{16}=-\frac{5}{72} .
$$

In the second case, see (22), the potential is expressed by

$$
v_{1}=\frac{91}{72}, \quad v_{2}=\ldots=v_{8}=-\frac{5}{72}, \quad v_{9}=\ldots=v_{16}=\frac{7}{72} .
$$

If we express the first Hamiltonian $H_{1}$, in equal blocks decomposition, as

$$
H_{1}=\left(\begin{array}{cc}
H_{B} & 0 \\
0 & H_{F}
\end{array}\right)
$$

the second Hamiltonian $\mathrm{H}_{2}$ is obtained by exchanging bosons with fermions through a similarity transformation induced by $\Gamma_{8}$ :

$$
H_{2}=\left(\begin{array}{cc}
H_{F} & 0 \\
0 & H_{B}
\end{array}\right)=\Gamma_{8} H_{1} \Gamma_{8}
$$

Any operator $g_{2}$ obtained from the $c=-\frac{1}{12}$ choice is related to an operator $g_{1}$ obtained from the $c=\frac{1}{12}$ choice via the similarity transformation

$$
g_{2}=\Gamma_{8} g_{1} \Gamma_{8}
$$

In the following, without loss of generality, we work with the choice of parameters given by (32).

The antisymmetry of $R_{I J}$ for $I \neq J$ implies that there are at most 28 generators defined by (28). One can nevertheless verify the existence, at the selected $c= \pm \frac{1}{12}$ values, of 7 linear constraints satisfied by the $R_{I J}$ 's. This results in a total number of 21 linearly independent generators. This is the number of $R$-symmetry generators entering the $F(4)$ superalgebra (whose $R$-symmetry is $s o(7)$ ).

Within the $c=-\frac{1}{12}$ choice the seven constraints aree covariantly expressed as

$$
2 R_{i 8}+C_{i j k} R_{j k}=0 .
$$

As a consequence of these relations, the 21 linearly independent generators can be accommodated into the rank 2 antisymmetric tensor $R_{i j}$.

We explicitly present for $c=-\frac{1}{12}$, in a covariant form, the non-vanishing (anti)commutators involving the $R$-symmetry generators $R_{i j}$. We have

$$
\begin{aligned}
{\left[R_{i j}, Q_{k}\right] } & =-\frac{i}{3} C_{i j k} Q_{8}+\frac{i}{3} C_{i j k l} Q_{l}+i \delta_{i k} Q_{j}-i \delta_{j k} Q_{i} \\
{\left[R_{i j}, Q_{8}\right] } & =\frac{i}{3} C_{i j k} Q_{k}
\end{aligned}
$$


and, similarly,

$$
\begin{aligned}
{\left[R_{i j}, \widetilde{Q}_{k}\right] } & =-\frac{i}{3} C_{i j k} \widetilde{Q}_{8}+\frac{i}{3} C_{i j k l} \widetilde{Q}_{l}+i \delta_{i k} \widetilde{Q}_{j}-i \delta_{j k} \widetilde{Q}_{i}, \\
{\left[R_{i j}, \widetilde{Q}_{8}\right] } & =\frac{i}{3} C_{i j k} \widetilde{Q}_{k} .
\end{aligned}
$$

Furthermore, after lengthy but straightforward computations, the $R$-symmetry commutators can be covariantly expressed as

$$
\begin{aligned}
{\left[R_{i j}, R_{k l}\right]=} & i\left(\delta_{i k} R_{j l}-\delta_{i l} R_{j k}-\delta_{j k} R_{i l}+\delta_{j l} R_{i k}\right)+ \\
& -\frac{i}{6}\left(\delta_{i k} C_{j l m n} R_{m n}-\delta_{i l} C_{j k m n} R_{m n}-\delta_{j k} C_{i l m n} R_{m n}+\delta_{j l} C_{i k m n} R_{m n}\right)+ \\
& \frac{i}{3}\left(C_{i j k m} R_{m l}-C_{i j l m} R_{m k}-C_{k l i m} R_{m j}+C_{k l j m} R_{m i}\right)+\frac{i}{3}\left(C_{i j m} C_{k l n} R_{m n}\right) .
\end{aligned}
$$

Despite the appearance in the right hand side of (39]40]41) of the rank 3 and rank 4 totally antisymmetric octonionic structure constants, the graded Jacobi identities are satisfied by construction.

The overall result here presented is a covariant realization, in terms of first-order and second-order matrix differential operators, of the exceptional Lie superalgebra $F(4)$, whose even sector is given by $s l(2) \oplus s o(7)$ and whose odd sector contains $2 \times 8=16$ odd generators. The operators $H, D, K$ close the $s l(2)$ subalgebra, with the dilatation operator $D$ being the Cartan's element. The $s o(7)$ subalgebra is realized by the $R_{i j}$ generators, while the odd sector is given by the operators $Q_{I}$ 's and $\widetilde{Q}_{I}$ 's.

\section{The exceptional $F(4)$ deformed oscillator}

In Section 4 we proved that the scale-invariant $\mathcal{N}=8$ supersymmetric Hamiltonians $H$ introduced in Section 3 become superconformal (with an $F(4)$ dynamical symmetry) at the critical values $c= \pm \frac{1}{12}$. The resulting superconformal Hamiltonian, up to similarity transformations, is unique.

We introduce now the analogue of the De Alfaro-Fubini-Furlan construction [1], presenting the (deformed) quantum oscillator possessing the exceptional Lie superalgebra $F(4)$ as its dynamical symmetry. The deformed oscillator Hamiltonian is given by the linear combination $H+K$, where $K$ is introduced in (26). $K$ adds the oscillator damping term to the Calogero potentials.

It is particularly rewarding to investigate the properties of the $H+K$ deformed oscillator Hamiltonian because it possesses a (degenerate) ground state with a discrete, bounded from below, spectrum.

Both Hamiltonians, $H$ and $H+K$, can be compactly written as

$$
H_{\epsilon}=-\frac{1}{2} \partial_{x}^{2} \mathbb{I}_{16}+\frac{1}{2} \epsilon x^{2} \mathbb{I}_{16}+\frac{1}{x^{2}} V, \quad V=\operatorname{diag}\left(v_{1}, v_{2}, \ldots, v_{16}\right),
$$

for

$$
v_{1}=\frac{91}{72}, \quad v_{2}=\ldots=v_{8}=-\frac{5}{72}, \quad v_{9}=\ldots=v_{16}=\frac{7}{72},
$$


in such a way that $H$ is recovered for $\epsilon=0$ and the deformed oscillator Hamiltonian $H+K$ is recovered for $\epsilon=1$.

Eight pairs of creation/annihilation operators $(I=, 1,2, \ldots, 7,8)$ are introduced through the positions

$$
a_{I}=Q_{I}-i \widetilde{Q}_{I}, \quad a_{I}^{\dagger}=Q_{I}+i \widetilde{Q}_{I}
$$

For later convenience we also introduce the symbol $\bar{I}=0,1, \ldots, 7$, identifying $a_{0}:=a_{8}, a_{0}^{\dagger}:=a_{8}^{\dagger}$ and $\Gamma_{0}:=\Gamma_{8}$.

The Hamiltonian $H_{\epsilon=1}$ is recovered, for any $\bar{I}$, from the anticommutators

$$
\frac{1}{2}\left\{a_{\bar{I}}, a_{\bar{I}}^{\dagger}\right\}=H_{\epsilon=1}
$$

The commutators give

$$
\left[a_{\bar{I}}, a_{\bar{I}}^{\dagger}\right]=\mathbb{I}_{16}+Y_{\bar{I}}
$$

where $Y_{\bar{I}}=-\left[E_{\bar{I}}, \Gamma_{\bar{I}} \Gamma_{9}\right]$ are constant and traceless diagonal matrices. We have

$$
\begin{gathered}
Y_{\bar{I}}=\operatorname{diag}\left(y_{1}, \ldots, y_{16}\right), \\
y_{1}=-\frac{7}{3}, \quad y_{2}=\ldots=y_{8}=\frac{1}{3}, \quad y_{9+\bar{J}}=-\frac{1}{3}+\frac{8}{3} \delta_{\overline{I J}} .
\end{gathered}
$$

$Y_{\bar{I}}$ anticommutes with both $a_{\bar{I}}$ and $a_{\bar{I}}^{\dagger}$ :

$$
\left\{a_{\bar{I}}, Y_{\bar{I}}\right\}=\left\{a_{\bar{I}}^{\dagger}, Y_{\bar{I}}\right\}=0
$$

Due to these properties, $a_{\bar{I}}\left(a_{\bar{I}}^{\dagger}\right)$ are annihilation (creation) operators such that

$$
\left[H_{\epsilon=1}, a_{\bar{I}}\right]=-a_{\bar{I}}, \quad\left[H_{\epsilon=1}, a_{\bar{I}}^{\dagger}\right]=a_{\bar{I}}^{\dagger} .
$$

Each $\bar{I}$ defines 16 lowest weight representations $\mid \lambda_{k}^{(\bar{I})}>(k=1,2, \ldots, 16)$ introduced by the condition

$$
a_{\bar{I}} \mid \lambda_{k}^{(\bar{I})}>=0
$$

Each $\mid \lambda_{k}^{(\bar{I})}>$ lowest weight vector is a 16-dimensional vector whose only non-vanishing component is in the $k$-th position.

We recall that a vector is bosonic (fermionic) if it is an eigenvector of the Fermion Parity Operator $\Gamma_{9}$ with eigenvalue $+1(-1)$. It is straightforward to show that the bosonic lowest weight vectors $\left|\lambda_{k}^{(\bar{I})}\right\rangle$, which are obtained for $k=1,2, \ldots, 8$, do not depend on $\bar{I}$. They are annihilated by all eight operators $a_{\bar{I}}$. The lowest weight bosonic wave functions are proportional to

$$
\begin{aligned}
& \mid \lambda_{1}^{(\bar{I})}>\propto\left(x^{-7 / 6} e^{-\frac{1}{2} x^{2}}, 0, \ldots, 0\right)^{T} \\
& \mid \lambda_{j}^{(\bar{I})}>\propto\left(\delta_{j r} x^{1 / 6} e^{-\frac{1}{2} x^{2}}\right)^{T} \quad(j=2, \ldots, 8 ; \quad r=1, \ldots, 16) .
\end{aligned}
$$


The picture is quite different for the fermionic lowest weight vectors $\mid \lambda_{k}^{(\bar{I})}>$ with $k=$ $9,10, \ldots, 16$. None of them is annihilated by all eight operators $a_{\bar{I}}$.

For $\bar{I}=0,1, \ldots, 7$, the fermionic lowest weight vector $\left|\lambda_{9+\bar{I}}^{(\bar{I})}\right\rangle$, proportional to,

$$
\mid \lambda_{9+\bar{I}}^{(\bar{I})}>\propto\left(\delta_{9+\bar{I}, r} x^{7 / 6} e^{-\frac{1}{2} x^{2}}\right)^{T}
$$

is only annihilated by the operator $a_{\bar{I}}$.

The fermionic lowest weight vectors $\left|\lambda_{9+\bar{I}}^{(\bar{J})}\right\rangle$, with $\bar{J} \neq \bar{I}$, proportional to

$$
\mid \lambda_{9+\bar{I}}^{(\bar{J})}>\propto\left(\delta_{9+\bar{I}, r} x^{-1 / 6} e^{-\frac{1}{2} x^{2}}\right)^{T}, \quad(\bar{J} \neq \bar{I})
$$

are annihilated by the seven operators $a_{\bar{J}}, \bar{J} \neq \bar{I}$, while $a_{I} \mid \lambda_{9+\bar{I}}^{(\bar{J})}>\neq 0$. It follows from (53) that $\left|\lambda_{9+\bar{I}}^{\left(\bar{J}_{1}\right)}\right\rangle=\mid \lambda_{9+\bar{I}}^{\left(\bar{J}_{2}\right)}>$ for any pair $\bar{J}_{1}, \bar{J}_{2} \neq \bar{I}$.

We have determined a total number of 24 ( 8 bosonic and 16 fermionic) different lowest weight vectors.

Their lowest weight energy $E_{k}^{(\bar{I})}$ is computed from the relation

$$
E_{k}^{(\bar{I})}=\frac{1}{2}<\lambda_{k}^{(\bar{I})}\left|\left\{a_{\bar{I}}, a_{\bar{I}}^{\dagger}\right\}\right| \lambda_{k}^{(\bar{I})}>=\frac{1}{2}<\lambda_{k}^{(\bar{I})}\left|\left[a_{\bar{I}}, a_{\bar{I}}^{\dagger}\right]\right| \lambda_{k}^{(\bar{I})}>=\frac{1}{2}+\frac{1}{2}<\lambda_{k}^{(\bar{I})}\left|Y_{\bar{I}}\right| \lambda_{k}^{(\bar{I})}>.
$$

We get

$$
\begin{array}{rlrl}
E_{1}^{(\bar{I})} & =-\frac{2}{3}, & \\
E_{k}^{(\bar{I})}=\frac{2}{3}, & k=2,3, \ldots, 8, \\
E_{9+\bar{J}}^{(\bar{I})}=\frac{1}{3}+\frac{4}{3} \delta_{\overline{J I}}, & \bar{J}=0,1, \ldots, 7 .
\end{array}
$$

We will see in the following the implication of these results for the construction of the Hilbert space associated with the $F(4)$ deformed oscillator.

The eight creation (annihilation) operators $a_{\bar{I}}^{\dagger}\left(a_{\bar{I}}\right)$ are all unitarily equivalent. This is implied by the existence of seven unitary matrices $U_{i}\left(U_{i} U_{i}^{\dagger}=U_{i} U_{i}^{\dagger}=\mathbb{I}_{16}\right)$ satisfying

$$
U_{i} \Gamma_{i} U_{i}^{\dagger}=\Gamma_{8}, \quad U_{i} E_{i} U_{i}^{\dagger}=E_{8}, \quad U_{i} Y_{i} U_{i}^{\dagger}=Y_{8}, \quad\left[U_{i}, V\right]=0 .
$$

In the above relations the repeated indices are not summed.

As a consequence of (56) we have, in particular,

$$
U_{i} a_{i} U_{i}^{\dagger}=a_{0}, \quad U_{i} a_{i}^{\dagger} U_{i}^{\dagger}=a_{0}^{\dagger}
$$

for any $i=1,2, \ldots, 7$.

An explicit expression for $U_{1}$ is given by

$$
\begin{aligned}
U_{1}= & E_{1,1}+E_{2,2}+E_{3,3}+E_{4,4}-E_{5,8}+E_{6,7}-E_{7,6}+E_{8,5}+ \\
& E_{9,10}-E_{10,9}+E_{11,12}-E_{12,11}+E_{13,13}+E_{14,14}+E_{15,15}+E_{16,16},
\end{aligned}
$$


where $E_{r, s}$ denotes the $16 \times 16$ matrix with entry 1 at the intersection of the $r$-th row with the $s$-th column and 0 otherwise.

Similar expressions exist in the remaining cases $(i=2, \ldots, 7)$. For simplicity they are not reported here.

As a corollary of the unitary relations (57), the same set of lowest weight energy values (one eigenvalue $\frac{2}{3}$, seven eigenvalues $-\frac{2}{3}$, seven eigenvalues $\frac{1}{3}$ and one eigenvalue $\frac{5}{3}$ ) is encountered for each annihilation operator $a_{\bar{I}}$.

\section{Hilbert space and quasi-nonassociativity}

We determine the Hilbert space and the spectrum of the system with $F(4)$ as spectrumgenerating superalgebra.

We point out that the bosonic lowest weight vector $\left|\lambda_{1}\right\rangle \equiv\left|\lambda_{1}^{(\bar{I})}\right\rangle$ (the $\bar{I}$ dependence is dropped since the same lowest weight vector is shared by all $\bar{I}$ 's) of energy $-\frac{2}{3}$, see (55), is not normalized as a square integrable function since its norm is negative.

Indeed the norm of the function $f(x)=x^{-\frac{7}{6}} e^{-\frac{1}{2} x^{2}}$ is computed as

$(f, f)=\int_{-\infty}^{+\infty}|f(x)|^{2} d x=\int_{-\infty}^{0}|f(x)|^{2} d x+\int_{0}^{+\infty}|f(x)|^{2} d x$.

By setting $y=-x$ for $x<0$, we get $\int_{-\infty}^{0}|f(x)|^{2} d x=-\int_{\infty}^{0}\left|(-y)^{-\frac{7}{6}} e^{-\frac{1}{2}} y^{2}\right|^{2} d y=$ $\left|(-1)^{-\frac{7}{6}}\right|^{2} \int_{0}^{\infty} y^{-\frac{7}{3}} e^{-y^{2}} d y$, so that $(f, f)=\left(\left|(-1)^{-\frac{7}{6}}\right|^{2}+1\right) \int_{0}^{\infty} x^{-\frac{7}{3}} e^{-x^{2}} d x$.

After the $t=x^{2}$ change of variable we get

$$
(f, f)=\frac{1}{2}\left(\left|(-1)^{-\frac{7}{6}}\right|^{2}+1\right) \int_{0}^{+\infty} t^{-\frac{5}{3}} e^{-t} d t=\frac{1}{2}\left(\left|(-1)^{-\frac{7}{6}}\right|^{2}+1\right) \Gamma\left(-\frac{2}{3}\right)<0 .
$$

The eight independent fermionic lowest weight vectors resulting from $\mid \lambda_{9+\bar{I}}^{(\bar{J})}>$, with $\bar{J} \neq \bar{I}$, have energy eigenvalue $+\frac{1}{3}=-\frac{2}{3}+1$. They correspond to the first excited states $a_{\bar{I}}^{\dagger} \mid \lambda_{1}>$. Contrary to $\left|\lambda_{1}\right\rangle$, their norm is positive. Nevertheless, they have to be excluded from a Hilbert space since, by applying the annihilation operator $a_{\bar{I}}$, we obtain the state $\mid \lambda_{1}>$ with negative norm: $a_{\bar{I}} a_{\bar{I}}^{\dagger}\left|\lambda_{1}>\propto\right| \lambda_{1}>$.

The lowest weight representation defined by $\mid \lambda_{1}>$ (which also includes the $\mid \lambda_{9+\bar{I}}^{(\bar{J})}>$, with $\bar{J} \neq \bar{I}$, vectors) does not define a Hilbert space because not all states are correctly normalized.

The Hilbert space induced by the $F(4)$ oscillator model is obtained by the direct sum of the remaining lowest weight representations.

It is convenient to rename as $b_{i}(i=1,2, \ldots, 7)$ the seven bosonic lowest weight vectors $\lambda_{i+1} \equiv \lambda_{i+1}^{\bar{I}}$ from (51) with positive energy eigenvalue $\frac{2}{3}$. The eight fermionic lowest weight vectors $\mid \lambda_{9+\bar{I}}^{(\bar{I})}>$ from (152), with energy eigenvalue $\frac{5}{3}=\frac{2}{3}+1$, are the first excited states obtained from the $b_{i}$ 's bosonic states.

The construction goes as follows. We note at first that the creation operators $a_{\bar{I}}^{\dagger}$ satisfy the superalgebra

$$
\begin{aligned}
\left\{a_{\bar{I}}^{\dagger}, a_{\bar{J}}^{\dagger}\right\} & =2 \delta_{\overline{I J}} Z \\
{\left[Z, a_{\bar{I}}^{\dagger}\right] } & =0
\end{aligned}
$$


which is formally equivalent to the $\mathcal{N}=8$ superalgebra (15) of the Supersymmetric Quantum Mechanics. It should be noted however that the operator $Z$, defined by the first equation of (60), is not the Hamiltonian, but a raising operator. The superalgebra (60) is an implementation of the concept of "soft supersymmetry" discussed in [1].

It is convenient to set $f_{i} \equiv \mid \lambda_{9+i}^{(i)}>$ and $f_{0} \equiv\left|\lambda_{9}^{(0)}\right\rangle$, so that we obtain

$$
\begin{aligned}
f_{i} & =a_{0}^{\dagger} b_{i}, \\
f_{0} & =-a_{1}^{\dagger} b_{1}=-a_{2}^{\dagger} b_{2}=\ldots=-a_{7}^{\dagger} b_{7}
\end{aligned}
$$

(for any $i, f_{0}=-a_{i}^{\dagger} b_{i}$ ).

The covariant relation

$$
a_{i}^{\dagger} b_{j}=C_{i j k} f_{k}=C_{i j k} a_{0}^{\dagger} b_{k}
$$

is satisfied.

The vector space spanned by the direct sum of the seven lowest weight representations induced by the $b_{i}$ 's vectors admits, by construction, normalized vectors. This Hilbert space corresponds to $\mathcal{L}^{2}(\mathbb{R})^{16}$, a 16-ple of $\mathcal{L}^{2}(\mathbb{R})$, the square-integrable functions on the real line.

Let us introduce the state $g_{0}$, given by

$$
g_{0}:=a_{0}^{\dagger} f_{0}
$$

Its energy eigenvalue is $\frac{8}{3}$. We collectively denote as $w_{r}, r=1,2, \ldots, 16$ (by setting $\left.w_{i}=b_{i}, w_{8}=f_{0}, w_{8+i}=f_{i}, w_{16}=g_{0}\right)$, the $(7 ; 8 ; 1)$ states $b_{i} ; f_{0}, f_{i} ; g_{0}$ of energy eigenvalues $\left(\frac{2}{3} ; \frac{5}{3} ; \frac{8}{3}\right)$, respectively. Let $E_{r}$ denotes the energy eigenvalue of $w_{r}$. The Hilbert space of the theory is spanned by the states

$$
Z^{n} w_{r}, \quad r=1, \ldots, 16, \quad n \in \mathbb{N}_{0},
$$

where the operator $Z$ has been introduced in (60).

The energy eigenvalue of $Z^{n} w_{r}$ is

$$
E_{n, r}=2 n+E_{r}
$$

The energy spectrum of the theory is

$$
\frac{2}{3}, \frac{5}{3}, \frac{8}{3}, \frac{11}{3}, \ldots,
$$

Apart the ground state (which is 7 times degenerate), each excited level is 8 times degenerate:

$$
(7,8,8,8, \ldots)
$$

The degenerate states of each energy level are accommodated into a representation of the so(7) $R$-symmetry subalgebra.

The operator $Z$ is introduced in (60) as square of the $a_{\frac{1}{I}}^{\dagger}$ operators. For this reason the knowedge of the action of the $a_{\frac{1}{I}}^{\dagger}$ operators on the $(7 ; 8 ; 1)$ states $w_{r}$ 's is sufficient to reconstruct the whole semi-infinite tower of $(7,8,8,8, \ldots)$ states. The states $w_{r}$ 's are 
accommodated into the $(7 ; 8 ; 1)$ supermultiplet of the $\mathcal{N}=8$ worldline supersymmetry, see [6]. This supermultiplet (contrary to, e.g., the $\mathcal{N}=8$ supermultiplets $(k ; 8 ; 8-k)$ with $k=$ $2,3,4,5,6)$ preserves the octonionic covariance of the $\mathcal{N}=8$ worldline supersymmetry, see 5 .

The deformed (via Calogero potential terms) quantum oscillator induced by the exceptional superconformal algebra $F(4)$ is a unique system (up to similarity transformations) which only exists at a critical value of the parameters. It can be characterized by the property of "quasi-nonassociativity". The meaning is that the model is determined by the octonionic structure constants. Indeed, the structure constants of the $F(4)$ dynamical symmetry superalgebra are expressed in terms of the rank 3 and rank 4 totally antisymmetric octonionic structure constants, see formulas (39,40,41). More than that, the "strange" rational coupling constants of the $\frac{1}{x^{2}}$ Calogero potential term in the superconformal Hamiltonian (42) are given by the diagonal matrix (43), which reads as

$$
V=\operatorname{diag}\left(\frac{91}{72}, \frac{-5}{72}, \frac{-5}{72}, \frac{-5}{72}, \frac{-5}{72}, \frac{-5}{72}, \frac{-5}{72}, \frac{-5}{72}, \frac{7}{72}, \frac{7}{72}, \frac{7}{72}, \frac{7}{72}, \frac{7}{72}, \frac{7}{72}, \frac{7}{72}, \frac{7}{72}\right) .
$$

The above values are derived from the octonionic structure constants $C_{i j k}$ through the formula

$$
V=\frac{1}{72}\left(\Gamma_{8}-\frac{1}{36} C_{i j k} \Gamma_{i} \Gamma_{j} \Gamma_{k}\right) C_{l m n} \Gamma_{l} \Gamma_{m} \Gamma_{n}
$$

The non-associativity of the octonions is encoded in the coupling constants of the $F(4)$ quantum oscillator.

It should be noted that the supertrace of $V$ is vanishing due to the relation

$$
\operatorname{str} V=\frac{1}{72}(91+7 \times(-5)-8 \times 7)=0 .
$$

\section{Conclusions}

The Hilbert space of the one-dimensional $F(4)$ deformed quantum oscillator is given by a 16-ple of square-integrable functions. The energy levels are quantized to be $\frac{2}{3}+\mathbb{N}_{0}$. The ground state of energy $\frac{2}{3}$ is 7 times degenerate, while the excited states are 8 times degenerate. The eight creation operators $a_{\bar{I}}^{\dagger}$ close the $\mathcal{N}=8$ worldline superalgebra (60).

Therefore, the states of the seminfinite tower $(7,8,8,8, \ldots)$ are interconnected by the worldline supersymmetry induced by the irreducible, see [6], $(7 ; 8 ; 1)$ supermultiplet.

The model is uniquely determined (up to similarity transformations). The associated superconformal quantum mechanics is obtained from the most general octonioniccovariant and scale-invariant $\mathcal{N}=8$ supersymmetric quantum mechanics at a critical value of enhanced $F(4)$ dynamical symmetry.

The non-trivial Calogero's coupling constants are expressed in terms of the octonionic structure constants.

It is worth mentioning that, besides the construction of the $F(4)$ model, other results have been obtained by our investigation using the octonionic-covariant approach. We excluded, at $\mathcal{N}=7$, the existence of an octonionic covariant superconformal quantum mechanics realized on 16 fields and with $G(3)$ as spectrum-generating superalgebra. Even 
if a priori possible, not such a model exists for any combination of the parameters allowed by octonionic-covariance.

The superalgebra $D(4,1) \approx \operatorname{osp}(8 \mid 2)$ is the spectrum-generating superalgebra of the undeformed system (all Calogero's coupling constants are set to zero) given by the direct sum of 16 ordinary one-dimensional oscillators. The details of the construction will be reported in a larger forthcoming paper devoted to a general derivation of spectrumgenerating superalgebras of (un)deformed quantum oscillators.

Introducing nonassociativity in physics and quantum mechanics is notoriously a tricky business. So far, the unique truly nonassociative quantum mechanical system we are aware of, based on the Jordan's formulation of quantum mechanics, was derived in 1934 by Jordan, von Neumann and Wigner [13] and further analyzed in [14]. The observables are accommodated in the $3 \times 3$ Hermitian octonionic matrices (the exceptional Albert algebra).

There are several examples where nonassociative structures are relegated into the nonobservable sector of a quantum theory. For instance, the associativity deficit of the twisted cocycle condition of quasi-Hopf algebra, defined in [15] and discussed in [16], is often realized in canonical quantization, see [17], as a phase when projected onto the Hilbert space of the theory. The phase is a unitary transformation which can be reabsorbed in the normalized ray vector.

A different type of nonassociativity is encountered in the $F(4)$ deformed quantum oscillator model here presented. The nonassociativity of the octonions is encoded, as "quasinonassociativity", in the Calogero coupling constants of the theory which are given, see formula (69), in terms of the octonionic structure constants. The existence of such a relation is a consequence of the model admitting an octonionic-covariant formulation.

\section{Acknowledgments}

Z.K. and F. T. are grateful to the Osaka Prefecture University for hospitality. This research was supported by CNPq under PQ Grant 306333/2013-9. N. A. is supported by the grants-in-aid from JSPS (Contract No. 26400209).

\section{References}

[1] V. de Alfaro, S. Fubini and G. Furlan, Nuovo Cimento A 34 (1976) 569.

[2] F. Calogero, J. Math. Phys. 10 (1969) 2191.

[3] V. G. Kac, Commun. Math. Phys. 53 (1977) 31.

[4] A. Sudbery, J. Math. Phys. 24 (1983) 1986.

[5] Z. Kuznetsova, M. Rojas and F. Toppan, JHEP 0603 (2006) 098; arXiv:hep-th/0511274.

[6] A. Pashnev and F. Toppan, J. Math. Phys. 42 (2001) 5257; arXiv:hep-th/0010135.

[7] F. Delduc and E. Ivanov, Phys. Lett. B 654 (2007) 200; arXiv:hep-th/0706.2472. 
[8] Z. Kuznetsova and F. Toppan, J. Math. Phys. 53 (2012) 043513; arXiv:1112.0995[hep-th].

[9] S. Khodaee and F. Toppan, J. Math. Phys. 53 (2012) 103518; arXiv:1208.3612[hepth].

[10] N. L. Holanda and F. Toppan, J. Math. Phys. 55 (2014) 061703; arXiv:1402.7298 [hepth].

[11] I. E. Cunha, N. L. Holanda and F. Toppan, Phys. Rev. D 96 (2017) 065014; arXiv:1610.07205[hep-th].

[12] J. Baez, Bull. Am. Math. Soc. 39 (2002) 145; arXiv:math/0105155.

[13] P. Jordan, J. von Neumann and E. P. Wigner, Ann. Math. 35 (1934) 29.

[14] M. Günaydin, C. Piron and H. Ruegg, Comm. Math. Phys. 61 (1978) 69.

[15] S. Majid, "Foundations of Quantum Group Theory", Cambridge Univ. Press (1995).

[16] D. Mylonas, P. Schupp and R. J. Szabo, J. Math. Phys. 55 (2014) 122301; arXiv:1312.1621[hep-th].

[17] I. Bakas and D. Lüst, JHEP 0401 (2014) 171; arXiv:1309.3172[hep-th]. 